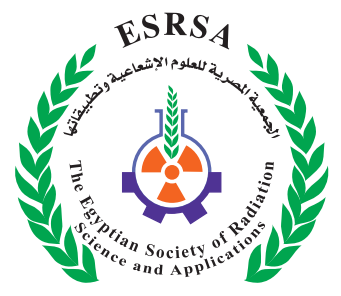

J. Nucl. Tech. Appl. Sci., Vol. 7, PP. 23 : 32 (2019)

\title{
Radiation Protection for Patients Undergoing Diagnostic Pelvic X-ray Examinations
}

Taha, T.M. ${ }^{1,2}$; Allehyani, S.H ${ }^{2}$; Alkhalaqi, $\mathrm{A}^{3}$ and Al-Kharouby, A. ${ }^{4}$

Received: 12/10/2018

Accepted: 09/12/2018

E.mail:tahaalfawwal@hotmail.com

\section{KEYWORDS}

Entrance Skin Dose, TLDs, Radiology, Gonad Shield, LLNL Phantom.

\section{ABSTRACT}

The aim of the current study is to measure the entrance skin dose (ESD) for patients undergoing pelvic diagnostic X-ray examinations in King Faisal Specialist Hospital (KFH), Saudi Arabia, and reduce the scattered $\mathrm{x}$-ray to gonads during the examination. The thermo-luminescence dosimeters (TLDs) are processed for low energy X-ray calibration. Three sets of thermo-luminescence dosimeters LiF (TLD-100) detector were irradiated by using a Xstrahl machine with following operating conditions: $60 \mathrm{~cm}$ FSD, $1 \mathrm{~mA}$, irradiation time 1 min., $2 \mathrm{~mm} \mathrm{HVL}(\mathrm{Al})$ and tube voltage of $80 \mathrm{kV}$. Each TLD set was irradiated for specific air and the mean value of the air kerma was used for calculation the calibration factor. The (ESD) was assessed for forty patients undergoing pelvic diagnostic X-ray examinations using thermo-luminescence dosimeters (TLDs). The patients were classified into three groups according to weights from $40-60 \mathrm{~kg}, 60-80 \mathrm{~kg}$ and $80-110 \mathrm{~kg}$ respectively. The ESDs associated with AP-pelvic examinations for the classified patients were $2.91 \pm 0.25$ $\mathrm{mGy}, 3.92 \pm 0.22 \mathrm{mGy}$ and $4.92 \pm 0.6 \mathrm{mGy}$ respectively. The results are compared with the reference levels of the European Union, (EU), International Atomic Energy Agency,(IAEA) and United Kingdom) . Another irradiation for TLD-100 chips was performed above and under the surface of the Lawrence Livermore National Laboratory phantom (LLNL, USA), and a scatter dose absorbed by sensitive organ attenuated by a factor of $20.6 \%$ due to using a flat contact gonadal shield .

1. Physics Department, Faculty of Applied Sciences, Umm Al-Quraa University, KSA.

2. Radiation Protection Department, Nuclear Research Center, Atomic Energy Authority, Cairo.P.O.13759 Egypt.,

3. Radiology department in Makkah Region, Department of Medical Physics.

4. Consultant pediatric radiologist, Head of Radiology department in Maternity and Children Hospital. 


\section{INTRODUCTION}

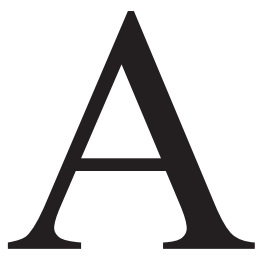

TLD is a phosphor, such as lithium fluoride that dopant by thallium , $\mathrm{LiF}(\mathrm{Tl})$ detector or calcium fluoride $(\mathrm{CaF})$, in a solid crystal structure. When a TLD is exposed to ionizing radiation at ambient temperatures, the radiation interacts with the phosphor crystal and deposits all or part of the incident energy in that material. Some of the atoms in the material that absorb that energy become ionized, producing free electrons and areas lacking one or more electrons, called holes. Imperfections in the crystal lattice structure act as sites where free electrons can become trapped and locked into place. Heating the crystal causes the crystal lattice to vibrate, releasing the trapped electrons in the process. Released electrons return to the original ground state, releasing the captured energy from ionization as light, hence the name thermo luminescent. Released light is counted using photomultiplier tubes and the number of photons counted is proportional to the quantity of radiation striking the phosphor. The amount of light released versus the heating of the individual pieces of thermo luminescent material is measured. TLD(s) dosimeters can be used for patient dosimetry external to the body or phantom in the same way as an ionization chamber are used for direct dose assessment (IAEA, 2014). Measurement of the (ESD for patients undergoing pelvic x-ray examinations should be performed in hospitals in order to use its results as a guideline for the optimization of the radiation protection of the patients. The ESD is a measure of the radiation dose that is absorbed by the skin as it reaches the patient, the radiation being in our case the X-ray beam used for the treatment of the patient. The $\mathrm{x}$-ray exposure in medicine field contributes significantly to about $95 \%$ total medical exposures. In Saudi Arabia, some work has been done for calculations of radiation dose to patients exposed in diagnostic radiology. Several calculations of the ESD were carried out for some routine X-ray examinations in the Security Forces Hospitals in Ri- yadh (Akhdar, 2007). Additionally, it has to be mentioned the work concerning the calculation of ESD for patients undergoing diagnostic x-ray carried out by (Taha et al., 2014) in King Abdullah Medical City, Makkah, Saudi Arabia and the effective dose calculation for the chest and abdomen performed by (Allehyani et al., 2015). The radio-sensitive organs such as gonads are susceptible of radiation risk due to the non-stochastic effects that can be expressed by a value of tissue weighting factor $\left(\mathrm{W}_{\mathrm{T}}\right)$ reported by the International Commission on Radiological Protection (ICRP-103, 2007). The ICRP reduced the tissue weighting factor of gonads from 0.2 to 0.08 . The present paper aims to assess the entrance skin dose to patients undergoing diagnostic pelvic $\mathrm{x}$-ray examinations in King Faisal Specialist Hospital (Saudi Arabia) and to minimize the scattered x-ray to gonads during the pelvix $x$-ray examination,

\section{MATERIALS AND METHODS}

The equipment used in this study includes an automatic TLD oven model PTW-TLDO (Physikalisch-Technische Workstation, Freiburg, Germany) , (TLD-100) of $\mathrm{LiF}(\mathrm{Tl})$ detector, Harshaw TLD ${ }^{\mathrm{TM}}$ Model 3500 Readers and two X-ray machines .First X-ray machine model Xstrahl -100 is used for irradiation of (TLD-100) of $\mathrm{LiF}(\mathrm{Tl})$ detector and examination the gonadal lead shield for decreasing scatter dose to radiosensitive organs. . Xstrahl -100 is adjusted for following operating conditions: $60 \mathrm{~cm}$ FSD, $1 \mathrm{~mA}$, irradiation time 1 min., $2 \mathrm{~mm} \mathrm{HVL(Al)}$ and tube voltage of $80 \mathrm{kV}$. The irradiation air kerma measured by Unidose meter connected with $0.2 \mathrm{cc}$ soft X-ray ionization chamber . the second X-ray machine manufactured by Siemens model Yasio , gemany manufacturer and model number 22814 is used for investigation of patients in radiology department in King Faisal Specialist Hospital (Saudi Arabia) and has the following features: automatic exposure control (AEC), fully digital flat detector technology that can used for flexible and versatile imaging in table, bulky wall stand (BWS) for free 
examinations and universal grid. The experimental arrangement for TLD dosimeter calibration and gonadal lead shield presented in figure 1(.a and b.) The procedure for assessment of entrance skin dose to patients includes three main parts, namely: (1) Calibration of of thermoluminscence dosimeters (TLDs); (2) distribution the (TLDs) on the skin of a patient in at the center of X-ray beam; (3) reading of the dosimeters by using a thermo-luminescence reader. Calibration of the thermo luminescence dosimeters (TLDs) includes several processes, as follows: pre-irradiation annealing, irradiation, sorting/ identification of the golden chips, pre-read annealing (preheating), irradiating- reading, and generation of the calibration factor.

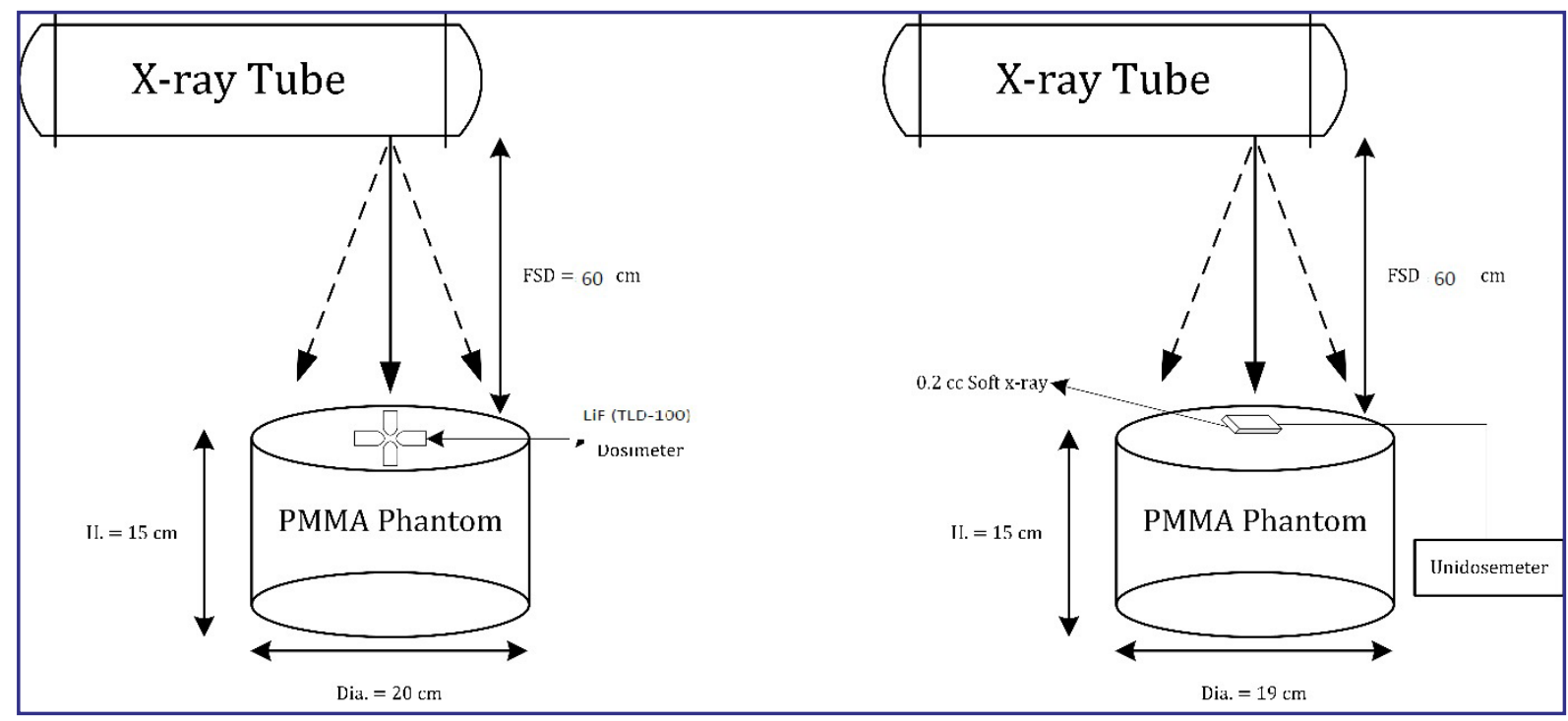

Fig. (1): The experimental arrangement for TLD dosimeter calibration.

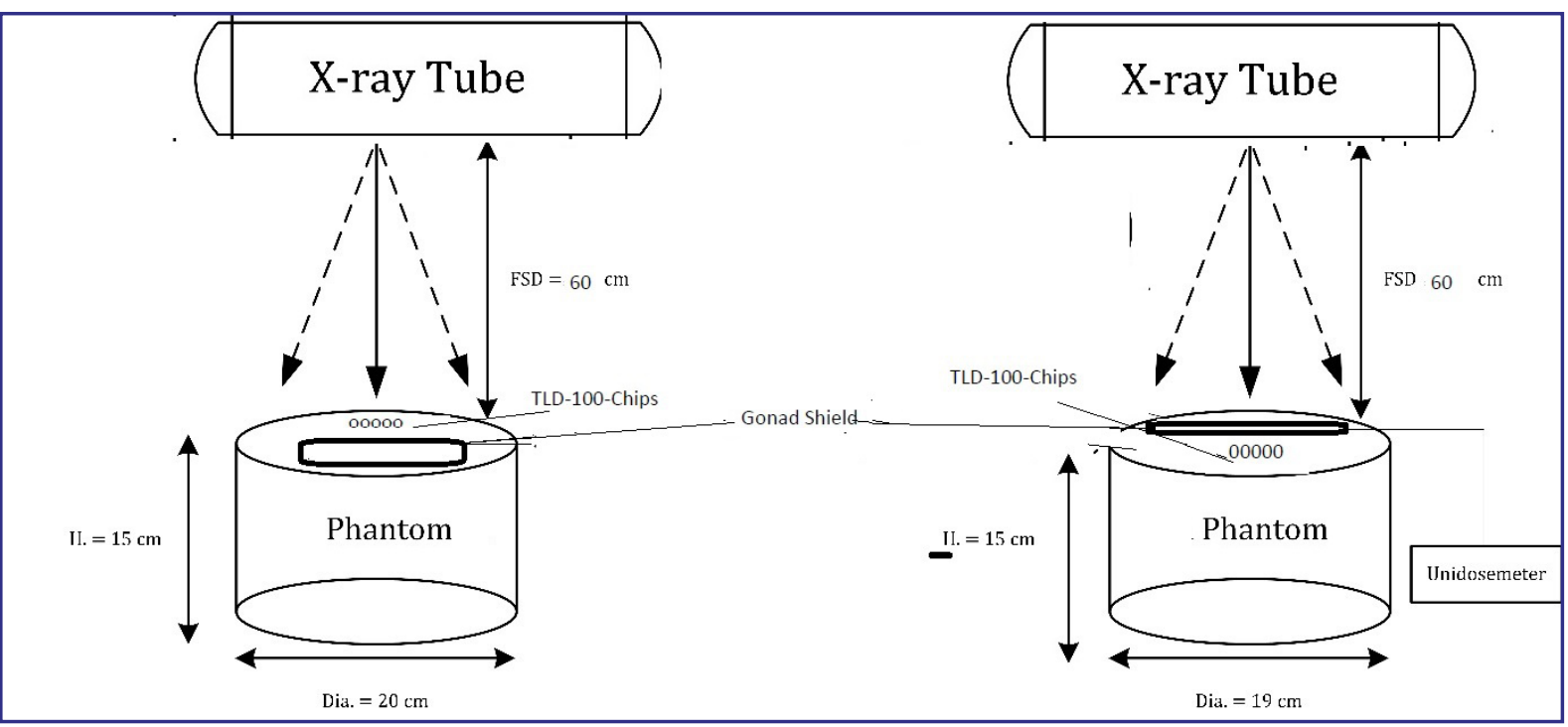

Fig. (2): The experimental arrangement for TLD dosimeter above and under gonad shield. 


\section{Irradiation}

Two batches of thirty TLD-100 each were annealed by using an automatic TLD oven model PTW-TLDO (Physikalisch-Technische Workstation, Freiburg, Germany). The pre-irradiation annealing procedure consisted of three steps, including high and low-temperature annealing according to the following sequence: 1 hour at $400{ }^{\circ} \mathrm{C}$, immediately followed by 2 hours at $100^{\circ} \mathrm{C}$, and finally 1 hour for cooling to room temperature. The TLD chips were irradiated with the x-ray generator. For each TL measurement by TLD-3500, reader background subtraction was performed. For each session of TLD exposure to radiation, up to 5 chips from the same group, with about the same sensitivity as those used for measurement, were set aside without being irradiated. This baseline group was subject to the same pre-readout procedure as the irradiated TLDs (Abulfaraj et al., 2004). TLD technique characterized by high precision and reproducibility of dose measurement is presented by addressing pre-readout annealing, group sorting, dose evaluation. Two hundred and forty TLD chips were annealed for 1 hour at $400^{\circ} \mathrm{C}$ followed by $2 \mathrm{~h}$ at $100^{\circ} \mathrm{C}$. After exposure of $1 \mathrm{mGy}$ air kerma from X-ray .irradiator TLDs were subjected to pre-readout annealing at $100^{\circ} \mathrm{C}$, then readout, sorted into groups each with nearly equal sensitivity, golden ships. The golden chips are defined as response for each dosimeters comparable to an average response of designed group of the dosimeters maintained as calibration dosimeters. TLD-100 chips have almost the same sensitivity, within $\pm 3 \%$, corresponding to standard deviation below $2.5 \%$. These chips are identified as the master to chips be used for the purpose of Dose Conversion Factor (DCF) generation. Upon repeating the procedures, TLDs having response $>3.5 \%$ from group mean were dropped to assuring group stability. Effect of pre-readout annealing has been studied. Series of repeated measurements were conducted to stabilize calibration procedures and DCF generation using standard X-ray machine.

\section{Identification of the golden chips}

It identified by careful analysis of the irradiated chips. TLD chips were readout within $24 \mathrm{hrs}$ of irradiation and sorted 50 TLD chips. In the first step, 30 chips were chosen from batch of the total number of 50 chips. After irradiation, the readout session was carried out in an uninterrupted manner. After readout, each TLD chip was kept in an assigned bin with the identification number for sorting purpose. This was done based on their statistical variation of recorded nC. Out of the 30 TLDs, the chosen group of 20 chips is representing the largest group in the statistical distribution having standard deviation below $10 \%$, and the chips were identified as master chips. In the second step, from these 20 master chips, 10 chips were identified with almost the same sensitivity, within $\pm 3 \%$, corresponding to standard deviation below $2.5 \%$. These chips were identified as the golden chips to be used for the purpose of Dose Conversion Factor (DCF) generation.

\section{Pre-read annealing (Preheating)}

A simplified TLD annealing technique (Luxton, 1999) characterized by high precision and reproducibility of radiation dose measurement was followed for the pre-read annealing process. In this technique, the TLDs are annealed, sorted, and subjected to pre-readout annealing according to the procedure described as follows: high temperature and lowtemperature annealing prior to irradiation, and prereadout annealing at low temperature after irradiation. High-temperature annealing was accomplished as mentioned before, using the PTW-TLD-Oven microprocessor controlled furnace, at $400{ }^{\circ} \mathrm{C}$ for 1 hour, followed by a low-temperature annealing at $100{ }^{\circ} \mathrm{C}$ for 2 hours, and pre-readout heating at 100 ${ }^{0} \mathrm{C}$ for two hours.

\section{Generation of reader calibration factor}

The numerical results obtained from the interpretation of the entrance dose assessment for patients exposed to external radiations depend mainly 
on the accurate calibration of the radiation measurement instrument used by investigators. This can be done by the conversion of $\mathrm{nC}$ obtained by heating the TL chips to equivalent dose (in mGy) which has been designated by Dose Conversion Factor (DCF). Unidose Dose master, (PTW -manufacture) coupled with a $0.2 \mathrm{cc}$ ion chamber located at a surface of LLNL's chest phantom, has been used for the reference doses measurement. These reference doses were carried out using the X-ray generator with an optimum operating conditions of kilo-voltage, miliamper second, and a suitable filter. The doses assessment was evaluated using the calibration factor that is based on X-ray energy, in $\mathrm{mGy} / \mathrm{nC}$.

\section{Sample distribution}

In the current study, the entrance surface dose (ESD) was determined for each patient undergoing radiographic pelvic examinations. The ESD was measured using thermo-luminescent dosimeters (TLDs) placed on the patient's skin, at the center of
X-ray beam, and after exposure, the readings were taken using a calibrated Harshaw TLD reader -3500.

\section{Gonadal shield attenuation}

Two TLD(s) chips sets were annealed, sorted, and irradiated using X-ray generator operating conditions similar to those of X-ray pelvic examination. One set was located at the surface of gonad positions using Lawrence Livermore National Laboratory's body phantom and the other set was located under the gonadal shield.

\section{RESULTS}

\section{Physical parameters of radiographic exposure data}

Physical parameters of the radiographic exposure data of X-ray exposure parameters for Pelvic AP projection, Peak kilovoltage $(\mathrm{kVp})$, Exposure time (in $\mathrm{mAs}$ ), Film-focus distance (FFD, in $\mathrm{cm}$ ), and Focus to skin distance (FSD, in $\mathrm{cm}$ ) are shown in table (1).

Table (1) : X-ray exposure parameters for Pelvic AP projection.

\begin{tabular}{|c|c|c|c|c|}
\hline Descriptive Value & KVp & mAs & FFD, cm & FSD, $\mathbf{c m}$ \\
\hline Max & 95 & 60 & 129 & 107 \\
\hline Min & 30 & 15 & 120 & 85 \\
\hline Mean & 76 & 30 & 120 & 96 \\
\hline StDev & 10 & 20 & 09 & 11 \\
\hline
\end{tabular}

\section{Data Collection}

The patients age was found to be in range from 14 to 80 years, weight was found to be in range from 40 to $109 \mathrm{~kg}$ and body mass index $\left(\mathrm{kg} / \mathrm{m}^{2}\right)$ was found to be in range from 5 to $49\left(\mathrm{~kg} / \mathrm{m}^{2}\right)$. Minimum and maximum value for patients age, weight and body mass index presented in (table.2).

\section{Generating reader calibration factor}

A sample of about 24 TLDs - 100 chips located inside a plastic sachet of $3 \mathrm{~mm}$ thickness and irradiated to $30.3 \mathrm{mGy}$ using Xstrah-100 X-ray machine. It was adjusted to the following operating conditions : x-ray at $80 \mathrm{kV}, 1 \mathrm{~mA}, 1 \mathrm{~min}$. exposure, filter number 4 and focal distance detector (FDD) of $50 \mathrm{~cm}$. 
The dose was measured using $0.2 \mathrm{cc}$ soft $\mathrm{x}$-ray connected with undoes meter (PTW manufacture). The calibration factor was found to be $0.075 \mathrm{mGy} / \mathrm{nC}$ and the coefficient of variation decreased from $5 \%$ to $2.5 \%$ by application of by application the protocol of American National Socity (ANSI,1993). Table (3) presents the calibration of TLD-data response, ranging from 376 to $411 \mathrm{nC}$, and the resulting calibration factor of $0.077 \pm 17 \mathrm{mGy} / \mathrm{nC}$.

Table (2) : Demographic data for the patients.

\begin{tabular}{|c|c|c|}
\hline Demographic Data & Descriptive Statistics & Value \\
\hline \multirow{2}{*}{ Age (years) } & Min & 14 \\
& Max & 80 \\
& Mean \pm SD & $43 \pm 19$ \\
\hline \multirow{2}{*}{ Weights $(\mathrm{kg})$} & Min & 40 \\
& Max & 90 \\
& Mean \pm SD & $66 \pm 19$ \\
\hline \multirow{2}{*}{ Body Mass Index $\left(\mathrm{kg} / \mathrm{m}^{2}\right)$} & Min & 5 \\
& Max & 49 \\
\hline
\end{tabular}

Table (3) : Generating Reader Calibration Factor, RCF.

\begin{tabular}{|c|c|c|c|c|}
\hline S.No & Reading $(\mathbf{n C})$ & S.No & Reading $(\mathbf{n C})$ & Cal. Factor, $\mathbf{m G y} / \mathbf{n C}$. \\
\hline 1 & 394 & 16 & 397 \\
\hline 2 & 410 & 17 & 377 \\
\hline 3 & 380 & 18 & 408 \\
\hline 4 & 415 & 19 & 398 \\
\hline 5 & 390 & 20 & 381 \\
\hline 6 & 405 & 21 & 405 \\
\hline 7 & 401 & 22 & 391 \\
\hline 8 & 406 & 23 & 390 \\
\hline 9 & 395 & 24 & 343 \\
\hline 10 & 401 & 25 & 416 \\
\hline 11 & 405 & 26 & 397 \\
\hline 12 & 394 & 27 & 377 \\
\hline 13 & 390 & 28 & 407 \\
\hline 14 & 343 & 29 & 381 \\
\hline 15 & 416 & 30 & 398 \\
\hline
\end{tabular}

A radiation calibration factor, $\mathrm{RCF}$, in $\mathrm{mGy} / \mathrm{nC}$, calculated using the following equation: $R C F=E C C X D s / T L s$ 
where $E C C$ is the element correction coefficient, $D_{s}$ is the assigned dose measured by $0.2 \mathrm{cc}$ ionization chamber connected with UNIDOS ${ }^{\text {webline }}$ Universal Dosimeter, $T L_{s}$ is the measured TLD signal in nano -Coulomb units (nC) after background correction.

\section{Analysis of entrance skin dose}

The investigated patients are classified into three intervals according to their weights, $40-60 \mathrm{~kg}$
, 60-80 kg and 80-110 $\mathrm{kg}$ respectively.

The mean and standard deviation values of ESD for pelvic $x$-ray examinations for each patient groups associated with AP-pelvic examinations were $2.95 \pm 0.23 \mathrm{mGy}, 3.49 \pm 0.22 \mathrm{mGy}$ and $4.98 \pm 0.6$ $\mathrm{mGy}$ respectively as presented in table 4 . The results of reference weight of 60-80 kg were compared with the those available from international publications as presented in (table 5).

Table (4): The ESDs associated with AP-pelvic examinations for three weight intervals and their corre sponding standard deviation.

\begin{tabular}{|c|c|c|}
\hline \multicolumn{3}{|c|}{ Entrance Skin Dose, ESD (mGy) } \\
\hline $40-60 \mathrm{~kg}$ & $60-80 \mathrm{~kg}$ & $80-110 \mathrm{~kg}$ \\
\hline 2.5 & 4.1 & 4.6 \\
\hline 3.1 & 3.9 & 5.9 \\
\hline 2.7 & 4.4 & 4.4 \\
\hline 3.1 & 3.8 & 5.2 \\
\hline 3.1 & 3.9 & 5.4 \\
\hline 3.1 & 4.1 & 5.2 \\
\hline 3.1 & 4.5 & 5.5 \\
\hline 3.1 & 3.8 & 5.4 \\
\hline 2.9 & 4.4 & 5.5 \\
\hline 2.6 & 3.8 & 5.4 \\
\hline 3.2 & 4.1 & 4.6 \\
\hline 2.5 & 3.8 & 4.2 \\
\hline 2.8 & 3.7 & 4.5 \\
\hline 3.2 & 3.6 & 4.3 \\
\hline 3.1 & 3.5 & 5.4 \\
\hline 2.8 & 3.7 & 5.0 \\
\hline 2.7 & 3.8 & 4.1 \\
\hline 2.9 & 3.6 & 4.5 \\
\hline 3.2 & 3.9 & 4.8 \\
\hline 2.5 & 4.1 & 4.7 \\
\hline \multicolumn{3}{|c|}{ Mean $\pm \sigma$} \\
\hline $2.95 \pm 0.23$ & $3.5 \pm 0.2$ & $4.98 \pm 0.57$ \\
\hline
\end{tabular}


Table (5): Mean of the ESDs ( $m G y)$ for pelvis examinations calculated for patients of reference weight of $70 \pm 10 \mathrm{~kg}$. compared with international organizations.

\begin{tabular}{|c|c|c|c|c|}
\hline X-ray projection & $\begin{array}{c}\text { Current work, } \\
\text { ESD, mGy }\end{array}$ & $\begin{array}{c}\text { (EC,1999) } \\
\text { mGy }\end{array}$ & $\begin{array}{c}\text { (IAEA,1996) } \\
\text { mGy }\end{array}$ & $\begin{array}{c}\text { (NRPB,2000) } \\
\text { mGy }\end{array}$ \\
\hline AP-Pelvic & $3.5 \pm 0.2(20)$ & 10 & 5 & 4 \\
\hline
\end{tabular}

\section{Radiation protection}

Radiation protection to Gonads during pelvic Xray examination. Ten TLD-100 dosimeters is distributed above and under $0.5 \mathrm{~mm}$ lead gonadal as presented in figure (1.b) . the percentage relative coefficient attenuation is presented in (table 6) . The percentage relative coefficient attenuation for gonadal shield is calculated according to the following equation. Af $=$ TLDf $/ \mathrm{TLDi}$

Where as Af : attenuation coefficient

$\mathrm{TLD}_{\mathrm{f}}$ : reading with gonadal lead shield

$\mathrm{TLD}_{\mathrm{i}}$ : reading without gonadal lead shield

Table (6) : TLD response without and with gonadal shield.

\begin{tabular}{|c|c|c|}
\hline $\begin{array}{c}\text { TLD response without } \\
\text { gonadal shield } \\
\text { nC }\end{array}$ & $\begin{array}{c}\text { TLD response with gonadal shield } \\
\text { nC }\end{array}$ & $\begin{array}{c}\text { Relative Attenaution coefficient } \\
\text { \% }\end{array}$ \\
\hline 300.7 & 69 & 22.9 \\
\hline 308.8 & 67 & 22.0 \\
\hline 289.2 & 75 & 25.9 \\
\hline 286.6 & 69 & 24.1 \\
\hline 298.6 & 80 & 26.8 \\
\hline 304.0 & 63 & 20.0 \\
\hline 308.2 & 63 & 20.4 \\
\hline 304.2 & 65 & 21. \\
\hline 308.2 & 70 & 23.0 \\
\hline 313.3 & 62.1 & 20.6 \\
\hline
\end{tabular}

The flat shield used to protect the gonads is made of lead, with a thickness of $0.5 \mathrm{~mm}$. When the shield was used, the intensity of the scattered radiation to gonads attenuated by a factor $20.6 \%$.

\section{DISCUSSION}

It can be seen in Table (1) that the mean value and standard deviation of tube voltage used for pelvic $\mathrm{x}$-ray examination was found to be $76 \pm 10 \mathrm{kVp}$.
The European Commission, (EC, 1999) recommended the use of tube voltage values of 100-120 $\mathrm{kVp}$ for adults. The range of tube voltage used for pelvic X-ray conventional radiography was within the operating conditions of the peak kilo-voltage settings. Table (3) presents the mean and standard deviation values of ESD for pelvic $\mathrm{x}$-ray examinations for three patient weights interval , 40-60 kg, 60-80 $\mathrm{kg}$ and 80-110 kg respectively. The ESDs associ- 
ated with AP-pelvic examinations were $2.95 \pm 0.23$ mGy , $3.49 \pm 0.22 \mathrm{mGy}$ and $4.98 \pm 0.6$ mGy respectively. The measured mean ESDs \pm standard deviation for the three patient weight groups are within the range of entrance skin dose carried out using TLD-100 and cited by the International Atomic Energy Agency, (IAEA-cn-85-142) .Table (4) presents the ESDs for patients of a reference weight of $70 \pm 10 \% \mathrm{~kg}$. It can be seen that the ESD (mGy) for AP pelvic x-ray examination decreased by $65 \%$ comparatively with the reference dose recorded by the European Commission (EC, 1996), by 30\% from the reference dose reported by International Atomic Energy Agency (IAEA, 1996) ,and by $12.5 \%$ from the reference dose given by the National Radiological Protection Board of United Kingdom (NRPB, 2000). The measurement of the ESD for patients in $\mathrm{KFH}$ was lower than the value of the international organizations, (NRPB, 2000) and that values reported by (Alatta, 2018) too. In addition, the ESD value for Pelvic is less than 10 times lower than that reported by (Alghoul et al., 2017). The mean and standard deviation of the ESDs received by patients of a reference weight of $70 \pm 10 \% \mathrm{~kg}$ and weights in range $40-60 \mathrm{~kg}$ and $80-110 \mathrm{~kg}$ in KFH Hospital were within the mean and standard deviation doses measured by thermoluminscence dosimetes which reported by (Begum, 1999). Table (5) presented the gonadal shield effectiveness by taken into account the ratio factor between TLD-response with 0.5 $\mathrm{mm}$ lead gonadal shield and TLD-response without shield .Flat contact gonadal shield reduces the dose to sensitive organ of LLNL's phantom by a factor of 22.6. This is lowered than that percentage attenuation coefficient found by (Fauber, 2011). The reason could be due to difference in the beam field size between the two studies.

\section{CONCLUSION}

The mean and standard deviation of the ESDs for pelvic x-ray examinations received by patients of a reference weight of $70 \pm 10 \% \mathrm{~kg}$ and weights in range 40-60 $\mathrm{kg}$ and 80-110 $\mathrm{kg}$ in KFH Hospital were within reference doses levels. The results obtained in this study shown also that the using of a gonadal shield reduce the scattered $\mathrm{x}$-ray radiation dose to gonads by a factor of $20.6 \%$ during X-ray pelvic examination. This study minimized a dose delivered to gonads and verify the optimization principle.. It is mandatory advice for the technologist to use gonad shield made of $0.5 \mathrm{~mm}$ lead to assure the protection of gonads during Pelvic x-ray examination.

Acknowledgments: This work is supported by Radiology Department of King Faisal Specialist Hospital (KFH). Makkah City, KSA.

\section{Conflict of interest}

The authors declare that they have no conflict of interest.

\section{REFERENCES}

- Alghoul, A.; Abdalla, M.M. and Abubaker, H.M. (2017): Mathematical evaluation of entrance surface dose (ESD) for patients examined by diagnostic $\mathrm{x}$ rays. J. Sci.;1(1): 8 .

- Abulfaraj, W.H.; Bhuiyan, S.I.; Taha, T.M.; and Elmohr, E. (2004): Quality Assurance and Quality Control Studies on TLD Based Dosimetry Services by the University Radiation Safety Committee of King Abdulaziz University. HPS.King Abdulaziz University, Jeddah 21589: KSA

- Akhdar, H. (2007): Assessment of Entrance Skin Dose and Effective Dose of Some Examinations using Calculation Techniques, M.Sc thesis, KSU.

- Allehyani, S.H.; Taha, T.M.; and Kutbi, R.A. (2016): Effective dose evaluation for chest and abdomen. I.J.S.R., e 5(3): 420.

- American National Standard Institute, ANSI (1993): American National Standard for dosimetry personnel Dosimetry Performance, Criteria for testing, ANSI N13.11.

- Begum, Z. (1999): Entrance Surface Dose measure- 
ment for some of the Radiological Patients in Bangladesh. IAEA-cn-85-193.

- European Commission on Radiation Protection 109 (1999): Guidance on diagnostic reference level (DRLs). For medical exposures. Luxembourg: OOPEC. International Atomic Energy Agency :international basic standards for protection against an ionizing radiation and for the safety of radiation source,

- Fauber, T.L. (2011): Gonadal Shielding in Radiography: A Best Practice?. Radiol. Technol., 88(2): 127.

- International Atomic Energy Agency, IAEA (2014): Diagnostic Radiology Physics. A handbook for Teachers and Student. Vienna, IAEA.

- Alatta, N.O.; Ali, W.M.; Hassan, H.A.; Hasan, I.A.; Ibrahim, S.A.A., Ajbna, E.M., Omer M.A.;
Wilson, A.B.M. and Abdalmalik A. (2018): Patient Radiation Dose Assessment in Routine Pelvic x-Ray Examination in Selected Hospitals in Khartoum Stat. I.J.S.R., 7(5): 741.

- National Radiological Protection Board, NRPB. (2000): National protocol for patient dose measurements in Diagnostic Radiology. Chilton Didcot. UK: NRPB. Report of the working party of the institute of Physics Science in Medicine.

- Taha, T.M.; F.H.Al-Ghorabie, F.H.; Kutbi, R.A. and Saib, W.S. (2014): Assessment of entrance skin doses for patients undergoing diagnostic X-ray examinations in King Abdullah Medical City, Makkah, KSA. J. Rad. Res. Appl.Sci., 8(1): 100. 